\title{
Comportamento das pressões respiratórias máximas após um programa de fisioterapia respiratória em idosos institucionalizados
}

Behavior of maximal respiratory

pressures after a respiratory

therapy program in institutionalized

FisiSenectus. Unochapecó Ano 3, n. 1 - Jan/Jun. 2015 individuals

Marieli Slongo. marislongo10@hotmail.com

Fisioterapeuta e aluna egressa da Universidade Regional Integrada do Alto Uruguai e das

Missões - Erechim

Ana Lucia Bernardo de Carvalho Morsch. analuepm@yahoo.com.br

Mestre em Ciências da Saúde, docente do curso de fisioterapia da Universidade Regional

Integrada do Alto Uruguai e das Missões.

\section{Resumo}

Introdução: Durante o processo de envelhecimento somado à institucionalização, os músculos respiratórios sofrem alterações que podem levar ao comprometimento da função respiratória e consequente diminuição da força muscular e das capacidades pulmonares em indivíduos idosos. Devido a essas alterações, observou-se a necessidade e a importância de realizar um programa de fisioterapia respiratória para esses indivíduos, considerando que estes apresentam uma diminuição das atividades de vida diária que podem causar um decréscimo ainda maior da função pulmonar. Objetivos: Avaliar as pressões respiratórias máximas dos idosos institucionalizados em um asilo na cidade de Erechim, pré e pós um programa de fisioterapia respiratória. Materiais e Métodos: A amostra foi composta por 13 idosos, de ambos os sexos, com idade entre 65 a 85 anos. As pressões respiratórias máximas foram avaliadas pré e pós-realização de um protocolo de fisioterapia respiratória. Os indivíduos foram sorteados e divididos em dois grupos, o G1 utilizou inspirometria de incentivo e treinador muscular expiratório e o G2 realizou exercícios respiratórios. 0 protocolo foi aplicado durante 24 sessões, três vezes por semana, durante oito semanas. Resultados: O G2 apresentou um aumento estatisticamente significativo de PImáx e PEmáx $(p=0,01)$, já o $G 1$ não apresentou alterações significativas. Conclusão: 0 grupo que realizou fisioterapia com exercícios respiratórios apresentou um aumento das pressões respiratórias máximas em comparação ao G1, fato que pode ser explicado pelo tamanho reduzido da amostra.

\section{Palavras-chave}

Testes de função respiratória, Modalidades de Fisioterapia, Instituição de longa permanência para idosos. 


\begin{abstract}
Introduction: During the aging process coupled with the institutionalization, the respiratory muscles undergo changes that can lead to impairment of respiratory function and consequent decrease in muscle strength and lung capacity in elderly individuals. Because of these changes, there was the need and the importance of holding a respiratory therapy program for these individuals, knowing that they present a decrease in activities of daily living, may cause an even greater decline in lung function. Objective: To assess the maximum respiratory pressures before and after an respiratory therapy program of institutionalized elderly in a nursing home in the city of Erechim. Materials and Methods: The sample consisted of 13 elderly of both sexes aged 65-85 years. Maximal respiratory pressures were evaluated pre and post of a respiratory therapy protocol. Individuals were randomly selected and divided into two groups, $\mathrm{G} 1$ used incentive spirometry and expiratory muscle coach and G2 performed breathing exercises. The protocol was applied for 24 sessions three times per week for 8 weeks. Results: Group 2 showed a statistically significant increase in MIP and MEP $(p=0,01)$, but the G1 did not presented significantly change. Conclusion: The group that performed physical therapy through breathing exercises showed an increase in respiratory muscle strength compared to group 1 , which may be explained by the small sample size.
\end{abstract}

\title{
Keywords
}

Respiratory function tests, physical therapy modalities, homes for the aged.

\section{Introdução}

$\infty \infty \infty \times \infty \times \infty \times \infty \times \infty \times \infty \times \infty \times \infty \times \infty \times \infty \times \infty \infty$

Com o decorrer da idade, a probabilidade do idoso ser institucionalizado aumenta. A institucionalização leva o indivíduo a uma diminuição das atividades de lazer e de ocupação do tempo livre. As condições materiais também não contribuem para atividade cotidiana institucional porque há grande carência de equipamentos e de recursos materiais nas instituições. A limitação da independência física leva à incapacidade para cuidar da própria pessoa e para exercer outras atividades do dia a dia, levando, inclusive, à limitação da integração social, tornando o indivíduo incapaz de manter e participar normalmente das relações sociais. 0 sistema respiratório também sofre alterações inerentes ao processo de envelhecimento, como diminuição relativa da capacidade pulmonar e do volume expiratório forçado no primeiro segundo (VEF1), e o conhecimento dessas modificações auxilia na detecção e prevenção de distúrbios respiratórios em idosos ${ }^{1-2}$.

Além das alterações dos volumes e capacidade pulmonares, um estudo observou que a idade apresentou uma correlação negativa entre a Pressão Inspiratória Máxima (PI máx) e Pressão Expiratória Máxima (PE máx) dos indivíduos, isto é, quanto maior a idade, menores as pressões respiratórias ${ }^{3}$.

0 processo de envelhecimento está associado à redução da massa muscular diafragmática e acessória, ocorrendo um declínio na produção de trabalho respiratório para um mesmo nível de estimulação neural. 0 aumento da complacência do compartimento abdominal em indivíduos mais velhos pode dissipar a pressão gerada durante a expiração forçada, reduzindo a pressão estática máxima ${ }^{4}$. Ocorre uma moderada redução na capacidade pulmonar total e um aumento no volume residual com a idade. A PI máx e PE máx são geradas nos volumes pulmonares mais baixos e mais altos, respectivamente, e essas adaptações fisiológicas podem, também, contribuir com o declínio das pressões respiratórias máximas associadas à idade. Durante o processo de envelhecimento, os músculos respiratórios sofrem alterações, podendo levar ao comprometimento da função respiratória e consequente diminuição da força muscular e das capacidades pulmonares que podem acarretar complicações como: atelectasias, pneumonias, hipoxemia arterial, entre outras ${ }^{5}$.

Existem poucos estudos a respeito do ganho de força muscular inspiratória (PImáx), em um deles, as mensurações de pressões inspiratórias máximas, antes e após a terapêutica por inspirômetro de incentivo, sofreram um 
acréscimo de aproximadamente $8 \%$ em uma semana de treinamento ${ }^{6}$.

A inspirometria de incentivo é amplamente utilizada para expansão pulmonar e na prevenção de complicações pulmonares em adultos, crianças e idosos, porém, os efeitos da inspirometria realizada com inspirômetro a volume e a fluxo nos volumes pulmonares, além da atividade da musculatura de idosos, ainda são pobremente estudados ${ }^{7-10}$. Comercialmente, há dois tipos de inspirômetro de incentivo: a volume e a fluxo, sendo que ambos encorajam o paciente a inspirar até a capacidade pulmonar total utilizando um dispositivo visual. Alguns autores observaram uma alteração de padrão ventilatório e ativação da musculatura com a utilização do inspirômetro a fluxo $0^{11-14}$. Parreira et $\mathrm{al}^{15} \mathrm{e}$ Paisani et $a^{16}$ mostraram que a inspirometria de incentivo a volume promove um aumento do volume pulmonar com uma grande participação abdominal em adultos.

Observou-se a importância de realizar um programa de fisioterapia respiratória para indivíduos idosos, visto que estes apresentam uma diminuição das atividades de vida diária, podendo causar um decréscimo ainda maior da função pulmonar.

O objetivo principal deste estudo foi avaliar as pressões respiratórias máximas (PRM) dos idosos institucionalizados no Asilo Jacinto Godoy na cidade de Erechim, pré e pós um programa de fisioterapia respiratória.

Por meio dos objetivos específicos pretendeuse analisar um provável incremento das pressões inspiratória e expiratória máximas após a realização de um programa de fisioterapia respiratória, avaliar a função pulmonar préprograma de fisioterapia respiratória e verificar alterações da frequência cardíaca, saturação periférica de oxigênio, frequência respiratória e pressão arterial após cada sessão do programa.

\section{Materiais e métodos}

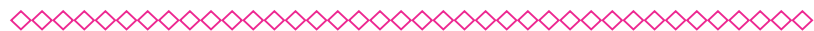

O presente estudo é de caráter observacional e descritivo de corte transversal. A amostra avaliada foi do tipo intencional, composta por 15 idosos, com idade entre 60 a 85 anos, residentes no Asilo Jacinto Godoy, localizado na avenida Sete de Setembro, 2141 na cidade de Erechim. 0 estudo foi aprovado pelo Comitê de Ética e Pesquisa da Universidade Regional Integrada do Alto Uruguai e das Missões - URI - Campus de Erechim/RS, sob o registro 186/TCH/07. Os objetivos, a metodologia da pesquisa e o Termo de Consentimento Livre e Esclarecido (TCLE) foram explanados para cada indivíduo. A partir da concordância dos idosos ou dos responsáveis na participação do estudo deuse início à coleta de dados.

Os critérios de inclusão foram: aceitação do TCLE pelos idosos ou responsáveis; idosos com idade entre 60 e 85 anos de ambos os sexos; idosos que consiguiam exercer suas atividades de vida diária; idosos aptos a realizar as atividades propostas. Como critérios de exclusão: idosos com doença obstrutiva crônica das vias aéreas; idosos com doença neurológica e idosos portadores de cardiopatia descompensada, constatada pelo médico com evolução no prontuário.

A coleta de dados foi composta por uma ficha de avaliação, onde constaram itens como, idade, sexo, hábitos de vida (tabagismo, prática de atividades físicas), presença de patologias, medicação e se já realizou fisioterapia. Nessa ficha foram registrados os resultados obtidos das avaliações pré e pós-tratamento da PE máx e PI máx. As variáveis espirométricas contidas na ficha de avaliação, capacidade vital forçada (CVF), volume expiratório forçado no primeiro segundo (VEF1), relação entre CVF/VEF1 e pico de fluxo expiratório (PEF) foram analisadas somente no início do tratamento para verificação da função pulmonar, e a frequência cardíaca ( $F C$ ) frequência respiratória (FR), pressão arterial (PA) e a saturação periférica de oxigênio (SpO2) foram verificadas no início e término de cada sessão.

Os indivíduos foram sorteados para participar dos grupos de fisioterapia respiratória, sendo que o grupo 1 (G1), composto por sete indivíduos, realizou um programa de fisioterapia respiratória com a utilização de recursos fisioterapêuticos Voldyne ${ }^{\circledR}$ e Threshold PEP ${ }^{\circledR}$ com $7 \mathrm{cmH} 2 \mathrm{O}$, e o grupo 2 (G2), composto por oito indivíduos, realizou um programa de fisioterapia respiratória 
com exercícios respiratórios compostos por padrões respiratórios.

O programa foi realizado três vezes por semana durante dois meses consecutivos com duração mínima de quarenta minutos a sessão. Para a avaliação da função pulmonar, foi utilizado o espirômetro Espirolab II da Micromedical, com valores preditos conforme a Sociedade Brasileira de Pneumologia e Tisiologia ${ }^{8}$.

Os indivíduos foram instruídos a realizar uma inspiração profunda até encher plenamente os pulmões e, em seguida, realizar uma expiração rápida, soprando todo o volume de ar contido. Essa expiração rápida e forçada foi mantida pelo maior tempo possível. 0 teste foi realizado com o paciente sentado em uma cadeira, com os braços ao longo do corpo e membros inferiores a $90^{\circ}$ de flexão, mantendo o nariz clampeado, sendo que todos os indivíduos realizaram no mínimo três vezes o teste e estes foram reprodutíveis e aceitáveis.

Para obtenção dos valores das pressões respiratórias máximas, PI máx (pressão inspiratória máxima) e PE máx (pressão expiratória máxima), verificadas no início e ao término do programa, foram seguidas as recomendações das Diretrizes das Provas de Função Pulmonar, conforme publicação do Jornal de Pneumologia (2002) ${ }^{17}$. Foi utilizado um manovacuômetro digital modelo MVD 30, marca Globalmed, com sistema de autocalibragem.

Durante a avaliação, os indivíduos permaneceram sentados, estando o tronco em um ângulo de $90^{\circ}$ com os MMII, e as narinas ocluídas por um clipe nasal. As manobras de avaliação foram previamente demonstradas pela pesquisadora para os participantes da pesquisa. Foram utilizados bocais plásticos, previamente higienizados à realização dos testes.

A avaliação da PI máx, que é a maior pressão gerada durante uma inspiração forçada contra uma via aérea ocluída, foi realizada a partir do esforço inspiratório iniciado ao nível do volume residual (volume de gás contido nos pulmões, após uma expiração máxima). Os indivíduos expiraram até alcançar seu volume residual, imediatamente, conectou-se a boca ao bocal e efetuou-se um esforço inspiratório máximo. o comando verbal utilizado para facilitar a execução dessa manobra foi: "solte todo o ar e depois puxe o ar com toda força no bocal".

A avaliação da PE máx, que é a maior pressão que pode ser desenvolvida durante um esforço expiratório vigoroso contra uma via aérea ocluída, foi realizada durante o esforço expiratório iniciado ao nível da capacidade pulmonar total (volume de gás contido nos pulmões após uma inspiração máxima). Foi recomendada a realização de uma inspiração até alcançar sua capacidade pulmonar total e em seguida conectou-se a boca ao bocal e efetuou-se um esforço expiratório máximo. 0 comando verbal utilizado para facilitar a execução dessa manobra foi: "puxe o ar até encher todo pulmão e depois sopre com toda força no bocal".

Cada indivíduo realizou cinco manobras para cada pressão avaliada, sendo que destas três deveriam ser aceitas, ou seja, não poderia haver vazamento de ar e a duração do esforço deveria ser mantida por pelo menos dois segundos. Entre cada manobra de avaliação das pressões respiratórias máximas foi dado um intervalo de 30 segundos e entre as avaliações de PI máx e PE máx foi dado intervalo de dois minutos.

Em outro momento foi dado início ao programa, sendo coletados, diariamente, FC, FR, PA e SpO2 ao início e término de cada sessão em ambos os grupos. A verificação da FC e da FR foi feita durante um minuto com o auxílio de um cronômetro. A pressão arterial foi aferida com esfigmomanômetro e estetoscópio da marca Premium, colocados no membro superior esquerdo do paciente. A SpO2 foi observada a partir de um oxímetro de marca Ohmeda, colocado no dedo indicador da mão do idoso.

Para a realização do programa de fisioterapia respiratória o grupo 1 (GI), que utilizou 0 recurso fisioterapêutico Voldyne, os indivíduos permaneciam sentados em uma cadeira com os MMII a $90^{\circ}$, segurando o aparelho em suas mãos, sendo o nariz clampeado com um clipe nasal. $\mathrm{Na}$ sequência, estes foram orientados a inspirar 0 ar pela boca com o bocal, sustentando o êmbolo do aparelho por dois segundos e soltando o ar lentamente pela boca fora do bocal. Essa técnica foi realizada em três séries de 15 repetições com intervalos de um minuto entre as séries. 
Para realizar o programa, foi utilizado o treinador muscular expiratório Threshold PEP, com $7 \mathrm{cmH2O}$. Nesse momento, os indivíduos permaneciam sentados em uma cadeira com os MMII a $90^{\circ}$, segurando o aparelho em suas mãos e foram orientados a colocar o clipe nasal sobre o nariz de forma que toda a respiração fosse feita pela boca e, após inspirar profundamente, deveriam expirar durante um tempo de duas a três vezes maior que na inspiração. A técnica foi realizada em três séries de 15 repetições com intervalos de um minuto entre as séries.

Os indivíduos do grupo 2 (G2) realizaram um programa composto de inspiração fracionada em três tempos associada à elevação dos membros superiores, expiração abreviada e padrão ventilatório com retardo expiratório.

Para a realização da inspiração fracionada em três tempos, os indivíduos permaneciam sentados e realizavam uma inspiração nasal, suave, curta, interrompida por curtos períodos de apneia pósinspiratória e programada para três tempos repetitivos associada à elevação de membros superiores (MMSS) concomitantemente, realizada em três séries de 15 repetições com intervalos de um minuto entre as séries.

Para realizar a expiração abreviada, que é composta por três fases, os indivíduos permaneciam sentados, sendo que na primeira fase era recomendado inspirar o ar pelo nariz de forma suave e profunda e em seguida expirar uma pequena quantidade de ar pela boca. Na segunda fase foram orientados a inspirar 0 ar pelo nariz profundamente a partir do término da primeira fase e, logo em seguida, expirar novamente uma pequena quantidade de ar pela boca. Na terceira fase os indivíduos foram instruídos a inspirar o ar profundamente pelo nariz a partir do término da segunda fase, soltando completamente o ar pela boca. Essa técnica foi realizada em três séries de quinze repetições com intervalos de um minuto entre as séries.

Para realizar o padrão ventilatório com retardo expiratório, os indivíduos também permaneciam sentados e foram orientados a inspirar profundamente o ar pelo nariz e, em seguida, expirar pela boca contra uma resistência que foi dada pelos dentes ou pelos lábios franzidos (soltando o ar como se pedisse silêncio). Essa técnica foi realizada em três séries de 15 repetições com intervalos de um minuto entre as séries.

Para analisar a variabilidade entre as variáveis dependentes da FC, SpO2, FR e PA antes e depois de cada sessão de fisioterapia respiratória e as medidas de PI máx e PE máx pré e pós-programa de fisioterapia respiratória, foi realizado o teste Wilcoxon, que é um teste não paramétrico alternativo para o teste t pareado, utilizando um software de estatística que analisa os resultados obtidos. Para a prova de função pulmonar avaliada pré-programa de fisioterapia respiratória somente foi aplicado o teste t para amostras de variâncias diferentes no teste de Wilcoxon.

\section{Resultados e discussão}

$\infty<\infty<\infty<\infty<\infty<\infty<\infty<\infty<\infty<\infty<\infty<\infty<\infty<\infty<$

A amostra foi composta por 15 idosos de ambos os sexos com idade entre 60 e 85 anos, avaliados no período de março a julho de 2008. Os participantes foram classificadas em dois grupos, sendo o grupo 1 (G1) composto por cinco idosos e o grupo 2 (G2) por oito idosos. O grupo 1 realizou um programa de fisioterapia respiratória com a utilização de Voldyne e Threshold PEP com $7 \mathrm{cmH} 2 \mathrm{O}$ e 0 grupo 2 realizou fisioterapia respiratória com exercícios respiratórios, utilizando respiração diafragmática com freno labial, inspiração fracionada em três tempos associada à elevação dos membros superiores e expiração abreviada. Dos sete idosos que faziam parte do G2, dois interoperam o tratamento, um devido a uma queda que ocasionou fratura de úmero e fêmur e o outro por desistência.

Foi aplicado o teste t para comparação entre as médias dos valores espirométricos obtidos do G1 e G2 pré-intervenção fisioterapêutica e pôde-se observar que não houve diferença estatisticamente significativa entre os grupos, conforme demonstrado na Tabela 1, ( $p>0,05)$.

Foi aplicado o teste de Wilcoxon para verificar se existe diferença significativa entre 0 valor previsto e as médias dos valores obtidos de cada uma das variáveis VEF1, CVF, CVF/VEF1 e PFE dos participantes da pesquisa. Pode-se 
observar, na Tabela 2 que não houve diferença estatisticamente significativa entre as variáveis, ( $p>0,05)$. Não houve diferença significativa entre o valor previsto e o valor medido de cada uma das variáveis VEF1, CVF, CVF/VEF1 e PFE nos participantes da pesquisa, isto é, os valores se enquadravam nos preditos para cada indivíduo.

A capacidade pulmonar total não diminui significativamente ao longo da vida. Isso ocorre porque a diminuição da complacência da parede torácica é contrabalançada pela maior distensibilidade pulmonar. A CVF e o VEF1 diminuem com a idade. Como fluxo é uma medida que reflete a relação de um dado volume em relação à unidade de tempo, temos, então, no indivíduo idoso, uma relação entre a força de contração e o tempo de resposta motora diminuídos. Além disso, a alta prevalência de doenças crônico-degenerativas no idoso, associada com a inatividade, faz com que esses indivíduos apresentem maior comprometimento da função pulmonar. O PFE também decresce com a idade em indivíduos idosos não fumantes, sugerindo que as alterações morfológicas das pequenas vias aéreas sejam decorrentes da presença de um padrão obstrutivo ${ }^{18}$.

Em outro momento foi verificado os sinais vitais e a saturação periférica de oxigênio, antes e após cada sessão de fisioterapia respiratória, com o objetivo de observar se estes se alterariam após os exercícios propostos. O teste de Wilcoxon foi aplicado nas diferenças das variáveis estudadas entre o pré e pós de cada sessão. Conforme mostra a Tabela 3, pode-se observar que não houve diferença estatisticamente significativa dessas variáveis para o $G 1,(p>0,05)$. A única diferença estatisticamente significativa foi na pressão arterial diastólica do $\mathrm{G} 2$, $(p<0,05)$.

No presente estudo observa-se que houve um declínio da pressão diastólica após as sessões de fisioterapia do G2, cujo programa consistia de exercícios respiratórios, tais como respiração diafragmática com freno labial, inspiração fracionada em três tempos associada à elevação dos membros superiores e expiração abreviada, porém, não se encontrou uma explicação para esse declínio.
Para comparar a diferença dos valores obtidos de PImáx e PEmáx pré e pós-realização do protocolo de fisioterapia respiratória proposto, ou seja, PI máx Pré - PImáx Pós e PEmáx Pré PEPós para os dois grupos, foi realizado o teste não paramétrico Wilcoxon. A Tabela 4 mostra que não houve diferença estatisticamente significativa dos valores obtidos para o G1 da PI máx e PE máx pré e pós-realização do protocolo de fisioterapia respiratória proposto $(p>0,05)$.

Em um estudo no qual 34 gerontes asilados foram divididos em dois grupos, grupo experimental (GE) e grupo controle (GC), cujo objetivo foi avaliar o efeito do fortalecimento muscular inspiratório sobre a PI máx e a autonomia funcional dos idosos asilados, comprovou-se que, no início do estudo, os grupos controle e experimental apresentaram valores de PI máx muito semelhantes GC: $31,67+11,11$ cmH2O $(p<0,05)$ e GE: $32,69+17,03 c m H 2 O(p<$ $0,05) 19$. No presente estudo foram encontrados valores de pressão inspiratória semelhante ao estudo mencionado.

Em um estudo comparativo sobre a alteração das forças inspiratórias e expiratórias entre jovens e idosos, utilizando o incentivador volumétrico Voldyne, participaram 18 indivíduos que foram divididos em dois grupos: grupo A (19 a 25 anos, de ambos os sexos) e grupo B (65 a 85 anos, de ambos os sexos, sedentários e sem doença pulmonar). Os indivíduos foram treinados por cinco semanas, nas quais realizavam três séries de dez repetições, três vezes por semana. Os resultados mostraram que o grupo A não conseguiu ganho de força (PI máx e PE máx). $O$ grupo $B$ obteve ganho significativo de força muscular inspiratória e expiratória. A pesquisa concluiu que a sustentação máxima inspiratória aumentou a força da musculatura respiratória dos idosos, o que não ocorreu com os jovens, visto que para estes haveria a necessidade de carga adicional devido à integridade do sistema pulmonar $^{15}$

Existem poucos estudos a respeito do ganho de força muscular inspiratória (PImáx) utilizando o Voldyne. Em um estudo com uso de incentivador, resultados demonstraram ganho de força dos músculos inspiratórios 
de aproximadamente $8 \%$ em uma semana de treinamento, sendo que os benefícios clínicos e terapêuticos da inspirometria de incentivo são: otimizar a insuflação pulmonar, os mecanismo da tosse e também da força muscular; detectar a doença pulmonar aguda mais cedo possível e melhorar a performance clínica do paciente em suas atividades de vida diária (AVD's) ${ }^{11}$.

0 aumento da pressão intra-alveolar é diretamente proporcional à força contrátil dos músculos respiratórios (diafragma e acessórios), justificando, assim, o fato de que para alcançar a capacidade pulmonar total (CPT) e sustentar a inspiração nesse nível ocorre uma intensa atividade muscular que é progressiva a partir do volume residual ${ }^{12}, 20,21$.

No atual estudo não houve incremento significativo de força inspiratória nos indivíduos que realizaram o protocolo de fisioterapia respiratória com o Voldyne. Tal resultado vai de encontro aos resultados obtidos em um estudo que comparou a utilização de inspirômetro de incentivo a fluxo e a volume, no qual, em relação às características do treinamento, ambos geraram baixa sobrecarga e, dessa forma, não se caracterizaram como treinamentos de força muscular ${ }^{22}$.

De acordo com o exposto, pode-se observar que houve diferença estatisticamente significativa na PI máx e PE máx do G2 ( $p<$ 0,05 ), que realizou o programa de fisioterapia respiratória com exercícios respiratórios compostos por respiração diafragmática com freno labial, inspiração fracionada em três tempos associada à elevação dos membros superiores e à expiração abreviada, como pode ser observada na Tabela 4.

Segundo alguns autores, os exercícios de membros superiores e o treinamento muscular respiratório podem melhorar a tolerância à dispneia, aumentar a força muscular respiratória e melhorar a qualidade de vida ${ }^{14},{ }^{20}$.

Os exercícios respiratórios promovem a aprendizagem de um padrão respiratório normal. Alguns autores relatam que a conscientização dos movimentos toracoabdominais gera ganho de força da musculatura respiratória. A realização de atividades físicas e metabólicas de forma satisfatória e equilibrada, com um gasto energético mínimo, favorece a reexpansão pulmonar, sendo facilmente visível o aumento da ventilação, da oxigenação e a melhora da mobilidade da caixa torácica. Além disso, os exercícios respiratórios por meio da inspiração fracionada podem trazer melhora na complacência pulmonar ${ }^{15},{ }^{16}, 22-24$.

O freno labial, por ser um exercício que promove uma resistência na expiração, pode ter contribuído no recrutamento dos músculos expiratórios, sendo que houve um aumento da PEmáx após a realização do programa. Os benefícios associados com o uso do padrão ventilatório com freno labial permitem ao paciente um melhor controle e conscientização sobre o trabalho ventilatório, reduzindo, assim, a sensação de dispneia, ocorrendo melhora na ventilação global e na coordenação muscular. Além disso, durante a fase expiratória, o retardo expiratório promove uma desinsuflação pulmonar homogênea, mantendo a pressão intrabrônquica e favorecendo, portanto, as trocas gasosas 25 .

No presente estudo foi realizado também o exercício respiratório diafragmático que permite uma maior expansão pulmonar, por aumento da ventilação nas bases, beneficiando aquelas na qual a complacência pulmonar esteja diminuída, aumentando também o volume pulmonar e melhorando as trocas gasosas. A respiração com freno labial diminui a frequência respiratória, aumenta o volume minuto e melhora a tolerância aos exercícios ${ }^{16},{ }^{18}$. Neste estudo não houve decréscimo da frequência respiratória, visto que os idosos não apresentavam desconforto respiratório e estavam estáveis.

0 incremento da força muscular respiratória aumenta a capacidade pulmonar a fim de compensar o aumento da carga respiratória, porém, ainda há vários pontos a serem questionados, tais como os mecanismos por meio dos quais esse tipo de treinamento melhora o desempenho no exercício incremental, bem como quais os dispositivos que resultam em maior eficiência ${ }^{26}$. 


\section{Considerações Finais}

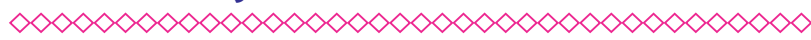

Houve incremento das pressões respiratórias máximas somente no grupo 2,0 que pode ser atribuído ao tamanho reduzido da amostra do grupo 1.

Mesmo que com poucas alterações encontradas após os programas de fisioterapia respiratória, é de grande importância a manutenção dos volumes e capacidades pulmonares e da força muscular respiratória para prevenir futuras desordens e/ou complicações inerentes ao processo de envelhecimento humano. Futuras pesquisas são necessárias devido à escassez de estudos nessa área.

\section{Referências}

$\infty \times \infty \times \infty \times \infty \times \infty \times \infty \times \infty \times \infty \times \infty \times \infty \times \infty \times \infty \times \infty$

1. Pegorari MS, Ruas G, Patrizzi LJ. Relationship between frailty and respiratory function in the community-dwelling elderly. Braz J Phys Ther. 2013 Jan-Feb;17(1):9-16.

2. Ruivo S, Viana P, Martins C, Baeta C. Efeito do envelhecimento cronológico na função pulmonar. Comparação da função respiratória entre adultos e idosos saudáveis. Rev Port Pneumol. 2009; XV (4): 629-653.

3. Neder JA, Andreoni S, Lerario MC, Nery LE. Reference values for lung function tests II. Maximal respiratory pressures and voluntary ventilation. Braz J Med Biol Res. 1999; 6(32):719727.

4. Britto RR, Vieira DSR, Rodrigues JM, Prado LF, Parreira VF. Comparação do padrão respiratório entre adultos e idosos saudáveis. Rev. Bras. Fisioter. 2005;9(3);281-287.

5. Simões RP, Castello V, Auad MA, Dionísio J, Mazzonetto M. Força muscular respiratória e sua relação com a idade em idosos de sessenta a noventa anos. RBCEH, Passo Fundo. 2010 jan/ $a b r ; 7(1): 52-61$.

6. Yamaguti WP, Sakamoto ET, Panazzolo

D, Peixoto CC, Cerri GG, Albuquerque AL.
Diaphragmatic mobility in healthy subjects during incentive spirometry with a flow-oriented device and with a volume-oriented device. J Bras Pneumol. 2010;36(6):738-745.

7. Restrepo RD, Wettstein R, Wittnebel L, Tracy M. AACR Clinical practice guideline: incentive spirometry: 2011. Respir Care. 2011; 56(10):16001604.

8. Gasparotto SC, Cardoso AL. Comparação do volume de ar obtido com duas formas diferentes de fluxo inspiratório durante exercício com incentivador. Fisioter Mov. 2009;22(3):355-363.

9. Dias CM, Vieira RO, Oliveira JF, Lopes AJ, Menezes SLS, Guimarães FS. Três protocolos fisioterapêuticos: efeitos sobre os volumes pulmonares após cirurgia cardíaca. J. Bras. Pneumol. 2011;37(1):54-60.

10. Carvalho CR, Paisani DM, Lunardi AC. Incentive spirometry in major surgeries: a systematic review. Rev Bras Fisioter. 2011;15(5):343-350.

11. Lunardi AC, Porras DC, Barbosa RCC, Paisani DM, da Silva CCBM, Tanaka C, Carvalho CRF. Effect of Volume-Oriented versus FlowOriented Incentive Spirometry on Chest Wall Volumes, Inspiratory Muscle Activity and Thoracoabdominal Synchrony in the Elderly. Resp Care. 2014;59(3):420-426.

12. Pascotini FS, Ramos MC; Silva AMV, Trevisan ME. Espirometria de incentivo a volume versus a fluxo sobre parâmetros respiratórios em idosos. Fisioter Pesq. 2013;20(4):355-360.

13. Chang AT, Palmer KR, McNaught J, Thomas PJ. Inspiratory flow rate, not type of incentive spirometry device, influences chest wall motion in healthy individuals. Physiother Theory Pract. 2010;26(6):385-392.

14. Parreira VF, Tomich GM, Britto RR, Sampaio RF. Assessment of tidal volume and thoracoabdominal motion using volume and flow-oriented incentive spirometers in healthy subjects. Braz J Med Biol Res. 2005; 38 (7): 110512. 
15. Parreira VF, Coelho EM, Tomich GM, Álvim AMA, Sampaio RF, Britto RR. Avaliação do volume corrente e da configuração toracoabdominal durante o uso de espirômetros de incentivo a volume e a fluxo, em sujeitos saudáveis: influência da posição corporal. Rev Bras Fisioter. 2004;8(1):45-51.

16. Paisani DD, Lunardi AC, da Silva CCBM, Porras DC, Tanaka C, Carvalho CRF. Volume Rather Than Flow Incentive Spirometry Is Effective in Improving Chest Wall Expansion and Abdominal Displacement Using Optoelectronic Plethysmography. Respir Care. 2013;58(8):13601366.

17. Pereira CAC. Diretrizes para testes de função pulmonar. J Pneumol. 2002; 28(03):S1-82.

18. Mayor A, Mayor UR. Avaliação funcional do aparelho respiratório aos efeitos do envelhecimento: aplicabilidade dos exercícios globais de força e resistência. Rev Fisioter Bras. 2004 jan/fev; $5(1): 56-60$.

19. Cader $S$, Silva EB, Vale $R$, Bacelar $S$, Monteiro MD, Dantas E. Efeito do treino dos músculos inspiratórios sobre a pressão inspiratória máxima e a autonomia funcional de idosos asilados. Motricidade. 2007;3(1):279-288.

20. Trevisan ME, Soares JC, Rondinel TZ. Efeitos de duas técnicas de incentivo respiratório na mobilidade toracoabdominal após cirurgia abdominal alta. Fisioter Pesq. 2010;17(4):322326.

21. Tomich GM, Franca DC, Diorio AC, Britto RR, Sampaio RF, Parreira VF. Breathing pattern, thoracoabdominal motion and muscular activity during three breathing exercises. Braz J Med Biol Res. 2007;40(10):1409-1417.

22. Reis IMM, Pessoa-Santos BV, Basso-Vanelli RP, Di Lorenzo VAP, Jamami M. Efeitos do treinamento com espirômetros de incentivo a fluxo e a volume em indivíduos saudáveis. R Bras Ci e Mov. 2015;23(2):104-112.

23. Vieira DSR, Mendes LPS, Elmiro NS, Velloso M, Britto RR, Parreira VF. Breathing exercises: influence on breathing pattern and thoracoabdominal motion in healthy subjects. Braz J Phys Ther. 2014 Nov-Dec;18(6):544-552.

24. Parreira VF, Bueno CJ, França DC, Vieira DS, Pereira DR, Britto RR. Breathing pattern and thoracoabdominal motion in healthy individuals: influence of age and sex. Rev Bras Fisioter. 2010;14(5):411-416.

25. Rossi RC, Pastre CM, Ramos EMC, Vanderlei LCM. A respiração frenolabial na doença pulmonar obstrutiva crônica: revisão da literatura. Fisioter. Pesq. 2012 july/sept;19(3).

26. Paiva DN, Assmann LB, Bordin DF, Gass R, Jost RT, Bernardo-Filho $M$, et al. Inspiratory muscle training with threshold or incentive spirometry: Which is the most effective? Rev Port Pneumol. 2014;21(2):76-81. 


\section{Anexos}

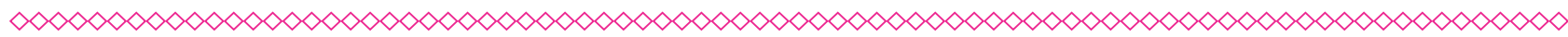

Tabela 1 - Características clínicas da amostra

\begin{tabular}{|c|c|c|c|}
\hline Variáveis Clínicas & G1 & G2 & p \\
\hline $\mathrm{N}$ & 5 & 8 &.. \\
\hline Idade (anos) & $74,2 \pm 7,6$ & $75,8 \pm 6,8$ &.. \\
\hline Sexo fem/masc & $2 / 3$ & $4 / 4$ &.. \\
\hline $\mathrm{VEF}_{1} \mathrm{CVF}_{\mathrm{CVF} / \mathrm{VEF}}$ & $2,11 \pm 0,31$ & $2,12 \pm 0,72$ & 0,491 \\
\hline $\mathrm{PFE}$ & $2,59 \pm 0,43$ & $2,79 \pm 0,79$ & 0,289 \\
\hline & $81,78 \pm 4,22$ & $75,55 \pm 8,47$ & 0,053 \\
\hline & $6,53 \pm 1,49$ & $5,35 \pm 2,29$ & 0,142 \\
\hline
\end{tabular}

Valores expressos em média e desvio padrão.

Tabela 2 - Variáveis espirométricas de ambos os grupos

\begin{tabular}{|c|c|c|c|c|}
\hline Variáveis & Previsto & Obtido & Dif & p \\
\hline VEF $_{1}$ & $2,33 \pm 0,72$ & $2,12 \pm 0,58$ & $0,21 \pm 0,53$ & 0,124 \\
\hline CVF & $2,99 \pm 0,98$ & $2,71 \pm 0,66$ & $0,28 \pm 0,63$ & 0,162 \\
\hline CVF/VEF & $74,30 \pm 1,07$ & $77,95 \pm 7,60$ & $-3,65 \pm 7,56$ & 0,108 \\
\hline PFE & $6,57 \pm 1,38$ & $5,80 \pm 2,04$ & $0,77 \pm 1,41$ & 0,108 \\
\hline
\end{tabular}

Valores expressos em média e desvio padrão, Dif: diferença entre os dados pré-intervenção de fisioterapia respiratória. 
Tabela 3 - Sinais vitais pré e pós-sessão do programa de fisioterapia respiratória dos grupos 1 e 2 .

\begin{tabular}{|c|c|c|c|c|}
\hline \multicolumn{5}{|c|}{ G1 } \\
\hline Variáveis & Pré & Pós & Dif (Pré - Pós) & p \\
\hline FC & $84,55 \pm 10,78$ & $83,44 \pm 8,62$ & $-1,11 \pm 2,23$ & 0,590 \\
\hline FR & $21,73 \pm 3,25$ & $21,04 \pm 2,37$ & $-0,69 \pm 1,16$ & 0,178 \\
\hline PA SIST & $135,70 \pm 12,99$ & $131,99 \pm 16,62$ & $-3,71 \pm 5,99$ & 0,281 \\
\hline PA DIAS & $81,87 \pm 2,69$ & $80,39 \pm 6,20$ & $-1,48 \pm 3,71$ & 0,281 \\
\hline SatO 2 & $93,72 \pm 1,60$ & $94,55 \pm 1,45$ & $0,83 \pm 0,37$ & 0,059 \\
\hline \multicolumn{5}{|c|}{ G2 } \\
\hline Variáveis & Pré & Pós & Dif (Pré - Pós) & p \\
\hline FC & $69,50 \pm 12,02$ & $71,10 \pm 10,46$ & $1,60 \pm 1,80$ & 0,059 \\
\hline FR & $19,35 \pm 0,93$ & $19,60 \pm 0,46$ & $0,24 \pm 0,66$ & 0,234 \\
\hline PA SIST & $122,09 \pm 8,12$ & $119,33 \pm 10,47$ & $-2,75 \pm 4,38$ & 0,234 \\
\hline PA DIAS & $79,35 \pm 2,82$ & $76,64 \pm 2,39$ & $-2,71 \pm 1,32$ & 0,014 * \\
\hline SatO 2 & $95,29 \pm 0,77$ & $95,35 \pm 0,91$ & $0,06 \pm 0,68$ & 0,889 \\
\hline
\end{tabular}

Valores expressos em média e desvio padrão, Dif: diferença entre os dados pré e pós-sessão de fisioterapia respiratória; ${ }^{*} \mathrm{p}<0,05$.

Tabela 4: Pressões respiratórias máximas pré e pós-programa de fisioterapia respiratória de G1 e G2.

\begin{tabular}{|c|c|c|c|c|c|}
\hline \multicolumn{5}{|c|}{ G1 } \\
\hline Variáveis & Pré & Pós & Dif (Pré-Pós) & Mediana (Dif) & p * \\
\hline PI máx & $34,80 \pm 15,61$ & $48,20 \pm 24,68$ & $3,4 \pm 16,51$ & 8,0 & 0,059 \\
\hline PE máx & $76,40 \pm 23,29$ & $101,40 \pm 23,26$ & $25 \pm 36,33$ & 11,5 & 0,059 \\
\hline \multicolumn{7}{|c|}{ G2 } \\
\hline Variáveis & Pré & Pós & Dif (Pré-Pós) & Mediana (Dif) & p * \\
\hline PI máx & $34,13 \pm 14,00$ & $44,00 \pm 16,97$ & $9,88 \pm 6,96$ & 9.250 & 0,014 \\
\hline PE máx & $83,00 \pm 24,73$ & $104,25 \pm 29,01$ & $21,25 \pm 16,16$ & 22.00 & 0,014 \\
\hline
\end{tabular}

Valores expressos em média e desvio padrão, Dif: diferença entre os dados pré e pós-prática de fisioterapia respiratória. A mediana corresponde aos valores das diferenças entre os dados pré e pósprática; * $p<0,05$. 